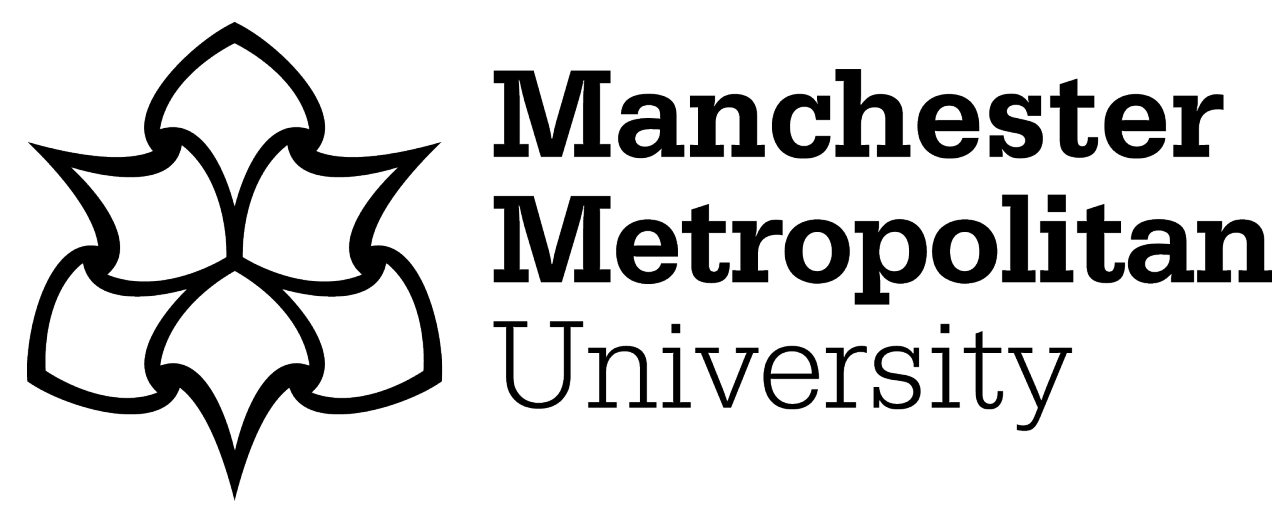

Trotter, PD, McGlone, F, McKie, S, McFarquhar, M, Elliott, R, Walker, SC and Deakin, JFW (2016) Effects of acute tryptophan depletion on central processing of CT-targeted and discriminatory touch in humans. European Journal of Neuroscience, 44 (4). pp. 2072-2083. ISSN 0953-816X

Downloaded from: https://e-space.mmu.ac.uk/618311/

Version: Accepted Version

Publisher: Wiley

DOI: https://doi.org/10.1111/ejn.13298

Please cite the published version 


\title{
Effects of acute tryptophan depletion on central processing of CT-targeted and discriminatory touch in humans
}

\author{
Paula Diane Trotter $^{1}$, Francis McGlone ${ }^{1,2}$, Shane McKie ${ }^{3}$, Martyn McFarquhar ${ }^{3}$, Rebecca \\ Elliott $^{3}$, Susannah Claire Walker ${ }^{1}$, John Francis William Deakin ${ }^{3}$ \\ ${ }^{1}$ Research Centre in Brain and Behaviour, School of Natural Sciences \& Psychology, \\ Liverpool John Moores University, Liverpool, UK \\ ${ }^{2}$ Institute of Psychology, Health and Society, University of Liverpool, Liverpool, UK \\ ${ }^{3}$ Neuroscience and Psychiatry Unit, The University of Manchester, Manchester, UK
}

Proposed journal section: Cognitive Neuroscience

Running title: Role of 5-HT in affective and discriminatory touch

Total pages: 30

Total Figures: 4

Total Tables: 4

Total equations: 0

Total words in whole manuscript: 10595

Total words in abstract: 250

Total words in introduction: 526

Keywords: Autism; CT afferents; Depression; functional Magnetic Resonance Imaging (fMRI); Serotonin (5-HT)

Address for correspondence: Dr Paula Trotter, School of Natural Sciences and Psychology, Tom Reilly Building, Liverpool John Moores University, Byrom Street, Liverpool, L3 3AF, UK, Email: $\underline{\text { P.D.Trotter@ljmu.ac.uk }}$ 


\section{Abstract}

C-tactile afferents (CTs) are slowly conducting nerve fibres, present only in hairy skin. They are optimally activated by slow, gentle stroking touch, such as those experienced during a caress. CT-stimulation activates affective processing brain regions, alluding to their role in affective touch perception. We tested a theory that CT-activating touch engages the pro-social functions of serotonin, by determining whether reducing serotonin, through acute tryptophan depletion, diminishes subjective pleasantness and affective brain responses to gentle touch.

A tryptophan depleting amino acid drink was administered to 16 healthy females, with a further 14 receiving a control drink. After 4 hours, participants underwent an fMRI scan, during which time CT-innervated forearm skin and CT non-innervated finger skin was stroked with 3 brushes of differing texture, at CT-optimal force and velocity. Pleasantness ratings were obtained post-scanning.

The control group showed a greater response in ipsilateral orbitofrontal cortex to CTactivating forearm touch compared to touch to the finger where CTs are absent. This differential response was not present in the tryptophan depleted group. This interaction effect was significant. Additionally, control participants showed a differential primary somatosensory cortex response to brush texture applied to the finger, a purely discriminatory touch response, which was not observed in the tryptophan depleted group. This interaction effect was also significant. Pleasantness ratings were comparable across treatment groups.

These results implicate serotonin in the differentiation between CT-activating and purely discriminatory touch responses. Such effects could contribute to some of the social abnormalities seen in psychiatric disorders associated with abnormal serotonin function. 


\section{Introduction}

Interpersonal touch promotes psychological well-being throughout the lifetime (Weiss et al., 2000; Feldman et al., 2010; Field, 2010; Burleson \& Davis, 2013) with lack of touch in childhood a significant predictor of adult depression (Brown et al., 2007; Takeuchi et al., 2010). Skin-to-skin contact has demonstrable clinical benefits for premature infants and those born full-term (Field, 2001; Bystrova et al., 2009; Moore et al., 2012; Feldman et al., 2014), and in adults, social touch can increase liking of a person or place, engender pro-social behaviours and increase trust (Morrison et al., 2010). However, little is known about the neurobiological mechanisms that transform touch stimuli to benefits on psychological development, resilience and well-being.

Numerous studies have demonstrated serotonin $(5-\mathrm{HT})$ is a key modulator of social responses with known effects on attachment formation and social bonding (Kiser et al., 2012; Young, 2013). Deakin \& Graeff (1991) hypothesised the interaction between social stimuli and serotonin is important in the pathogenesis of depression, proposing tactile interactions mediate the protective effects of close personal relationships. They cited rodent behavioural studies showing serotonergic drugs mimic group housing effects by preventing stress-induced anxietylike behaviour and the effects of isolation on serotonin function. More recent literature suggests hippocampal serotonin release mediates the long-term stress-protective effects of maternal care on rodent offspring (Meaney \& Szyf, 2005).

The recreational drug Ecstasy (3,4-methylene-dioxymethamphetamine (MDMA)) acutely increases central serotonin function (Morton, 2005) and is known to enhance the pleasure of touch (Klein et al., 2009). Conversely, experimental reduction of serotonin function following acute depletion of the essential amino acid and serotonin precursor, tryptophan (Delgado et al., 1990; Hood et al., 2005), has implicated serotonin in modulating tactile social cue perception in healthy volunteers (Bilderbeck et al., 2011; Bilderbeck et al., 2013). Taken together, these 
studies suggest serotonin modulates tactile encoding in general, and responses to socially relevant interactions in particular.

Two different classes of nerve fibre mediate cutaneous mechanosensation; large myelinated, fast conducting $A \beta$ afferents project to primary somatosensory cortex (SI) and encode discriminatory aspects of touch, whereas slow conducting, low-threshold mechanosensitive C-fibres (C-tactile afferents (CTs)) project to dorsal posterior insula and other limbic regions and are hypothesised to encode the affective components of gentle touch (Olausson et al., 2002, 2008b; McGlone et al., 2007, 2014). In humans, CTs are present in hairy skin, but have never been found in glabrous skin (Vallbo et al., 1999), and respond optimally to stroking velocities between $1-10 \mathrm{~cm} / \mathrm{s}$ delivered at human skin temperature (Loken et al., 2009; Ackerley et al., 2014); sensations experienced during a human caress. This evidence has led to the proposal that CTs are the neurobiological substrate of human social tactile behaviour (Morrison et al., 2010).

Deakin and Graeff (1991) proposed that social intimacy confers resilience to stress via an influence of affiliative touch in promoting serotonin function (Deakin, 1996). Here, we tested the prediction that if the CT system mediates affiliative touch, then reducing central serotonin function through acute tryptophan depletion (ATD) should reduce Blood Oxygenation Level Dependent (BOLD) responses specifically to $\mathrm{CT}$ activating touch in brain regions associated with affective processing. We also wished to determine whether ATD modifies discriminatory touch responses in somatosensory processing regions.

\section{Materials and methods}

\section{Ethics Statement}

Ethical approval was obtained from the North Manchester Research Ethics Committee. This experiment was undertaken with the understanding and written informed consent of each 
participant and the study conforms with the World Medical Association Declaration of Helsinki.

\section{Participants}

Thirty healthy female volunteers (mean \pm s.d. age $=23.7 \pm 5.18$ years) were recruited from the University of Manchester. All participants attended a screening session between 1 and 18 days (mean \pm s.d. $=4.6 \pm 3.3$ days) before the scanning session. Only female participants were included in this study to avoid the confound of sex on the data obtained. Females were chosen as they are almost twice as likely to be affected by depression than males (Hamet \& Tremblay, 2005) and are more susceptible to the effects of ATD (Nishizawa et al., 1997; Bell et al., 2005). Additionally, sex differences have been identified in responses to affective touch (Essick et al., 2010).

All participants had no self-reported psychiatric history and were physically healthy. The structured clinical interview to diagnose DSM-IV-TR Axis I disorders (SCID) (First et al., 2002) and the Brief Symptom Inventory (BSI) (Derogatis, 1993) were conducted during the screening session in order to exclude participants with a psychiatric history. Participants with average weekly alcohol consumption greater than 25 units per week were also excluded (mean \pm s.d. weekly alcohol consumption $=11.0 \pm 7.6$ units), as were participants who reported taking street drugs less than 4 weeks before participation. All participants were either taking the contraceptive pill, or tested during the follicular phase of their menstrual cycle. During screening, participants were provided with details of a low-protein diet to follow the day before the scan. Additionally, Intelligence Quotient (IQ) was measured using the Quick Test (Ammons \& Ammons, 1960) and touch ratings were completed as described in the following section, to allow comparison of ratings before and after amino acid drink consumption. 


\section{Touch Ratings}

All tactile stimuli were manually applied by the same female experimenter (PDT). Manual application of brush strokes to the forearm has been carried out in many previously published fMRI investigations of CT activating touch (Olausson et al., 2002, 2008a; Bjornsdotter et al., 2009; Morrison et al., 2011; Gordon et al., 2013; Voos et al., 2013; Kaiser et al., 2015). Apart from Morrison et al., (2011), these studies only used a soft brush, whereas this study used three brushes of varying degrees of coarseness. By varying stimulus texture, this enabled us to investigate whether central responses to CT-targeted touch were specifically tuned to soft, pleasant touch sensations. Brush strokes were applied to the ventral rather than dorsal forearm to enable access to the glabrous skin of the hand without requiring participants to move their arm.

The brushes used in this study were selected on the basis of a previous study where 16 participants, not included in the current study, provided Visual Analogue Scale (VAS) hedonic ratings of touch for a variety of brushes. These results were used to identify three brushes consistently rated as affectively pleasant (the soft brush), neutral (the medium brush) and unpleasant (the coarse brush) when applied to CT innervated forearm skin. None of the brushes were perceived as painful, nor produced any skin damage.

The three brushes selected for use in this study were all $44 \mathrm{~mm}$ wide, flat brushes and were as follows: soft - Daler-Rowney, 44 mm, Goat Hair, S155, Flat; medium - Hog Bristle, Daler-Rowney Georgian Brush, G36, Short Flat, no.18; coarse - plastic bristles with split ends mounted in the same flat handle as the soft brush.

For the current study, participants provided touch ratings of all brush stimuli during the screening session and immediately post scanning. The same experimenter (PDT) conducted all screening and scanning sessions. During the screening session, touch ratings were obtained after conducting the SCID (First et al., 2002) to determine psychiatric history. All tasks were 
completed by participants in the same order during the screening and scanning sessions, so all participants interacted with the experimenter a similar amount before providing touch ratings and experiencing the touch in the scanner.

Stimuli were applied in $20 \mathrm{~s}$ stimulation blocks preceded by a $20 \mathrm{~s}$ rest. During each stimulation block, participants were either stroked 5 times on their left mid ventral forearm over a distance of $18 \mathrm{~cm}$ in a proximal to distal direction, or 10 times on the ventral side of their left fingers, proximal to distal over $5 \mathrm{~cm}$, ending at the tip of the fingers. The interval between strokes was $1 \mathrm{~s}$. The experimenter maintained a CT optimal stroking velocity of 5 $\mathrm{cm} / \mathrm{s}$ (Loken et al., 2009), by synchronising the stroke with a moving dot on a monitor. The force of application was guided by the degree of bend in the brush, which was previously calibrated using a top pan balance to produce $220 \mathrm{mN}$. A touch-run consisted of one stimulation block per brush, applied to both the left forearm and fingers, in a randomised order. Randomisation of stimulus order each time the brush stimuli were administered removed systematic biases due to habituation to particular stimuli.

A single touch-run was used to obtain ratings during screening and after scanning. When providing touch ratings and when in the scanner, the left forearm of the participant was placed on a small VacFix ${ }^{\circledR}$ cushion for comfortable positioning of the arm. To avoid visual interference when providing touch ratings at screening and post scanning, participants wore a blindfold during stimulus application, which they removed to complete the VAS. Participants rated the pleasantness of each stimulus during the $20 \mathrm{~s}$ rest block following each stimulation block, on a $10 \mathrm{~cm}$ VAS with anchor points -10 (extremely unpleasant), -5 (unpleasant), 0 (neutral), 5 (pleasant) and 10 (extremely pleasant). One participant with aberrant ratings at screening was excluded from further participation.

The same computer programme written in E-prime version 2.0 (Psychology Software Tools, 2012) was used to guide stimulus application inside and outside the scanner, however, 
touch ratings were not taken during the scanning session. When in the scanner, the computer programme was projected on to the wall on the right hand side of the participant, so that it was visible to the experimenter, but not the participant.

\section{Imaging session}

\section{Amino acid drink}

ATD inhibits serotonin synthesis by reducing the availability of the essential amino acid and serotonin precursor, tryptophan. An amino acid load devoid of tryptophan is administered, inducing hepatic protein synthesis which depletes circulating tryptophan. Furthermore, the increase in large neutral amino acids competes with the transport of reduced levels of tryptophan across the blood-brain barrier via the large neutral amino acid transporter (Hood et al., 2005; Evers et al., 2010). The control condition is identical except the amino acid load contains tryptophan. This increases plasma tryptophan, but the ratio of tryptophan to other large neutral amino acids is still reduced, the reduction being significantly greater following ATD (Weltzin et al., 1994; Roiser et al., 2008).

Amino acids were supplied by SHS International Ltd. (Liverpool, UK). Participants were randomly assigned to receive either the tryptophan depleting (TRP-) drink or control drink (TRP+) and drinks were administered double blind. A between rather than within subject design limited the impact of participant withdrawal on the data. The ratio of amino acids used in the drinks was the same as that of Benkelfat et al. (1994), but $80 \%$ quantities were used to account for the lower average body weight of female participants (Hood et al., 2005). The amounts used are standard for ATD studies (e.g. Evers et al., 2006; Fusar-Poli et al., 2007; Bilderbeck et al., 2011; Daly et al., 2012, 2014). The total protein of the TRP- drink was 82.1 g. The amount of each amino acid contained in the TRP- drink was L-Alanine, $4.4 \mathrm{~g}$; LArginine 3.9 g; L-Cystine, 2.2 g; Glycine, 2.6 g; L-Histidine, 2.6 g; L-Isoleucine, 6.4 g; L- 
Leucine, 10.8 g; L-Lysine monohydrochloride, 8.8 g; L-Methionine, 2.4 g; L-Phenylalanine, 4.6 g; L-Proline, 9.8 g; L-Serine, 5.5 g; L-Threonine, 5.5 g; L-Tyrosine, 5.5 g; L-Valine, 7.1 g. The TRP+ drink was the same as the TRP- drink, with the addition of $1.8 \mathrm{~g}$ L-Tryptophan. A few minutes before oral administration, the amino acids were mixed with $150 \mathrm{ml}$ water and $\sim 45 \mathrm{ml}$ chocolate syrup to mask the unpleasant taste. Participants were required to consume the drink within 15 minutes. Immediately after drink consumption, participants completed questionnaires and cognitive tasks (not reported), then rested until the scanning session which began 4 hours after drink consumption.

Blood glucose and blood pressure were monitored throughout the day. Blood samples for commercial assay of total plasma tryptophan were taken before and 4 hours after the amino acid drink. Mood was monitored pre-drink and 4 hours post-drink using the Profile Of Mood States (POMS) (McNair \& Lorr, 1971) and the Fawcett-Clark Pleasure Scale (FCPS) (Fawcett et al., 1983).

\section{Scanning parameters}

Scanning was conducted using a Philips Achieva $3 \mathrm{~T}$ scanner. T2*-weighted functional images were obtained to investigate changes in BOLD signal throughout the scan. A single shot gradient echo-planar sequence was used. Whole brain scans of 34 slices, each $3 \mathrm{~mm}$ thick with a $0.5 \mathrm{~mm}$ slice gap, were obtained. The repetition time (TR) was $2000 \mathrm{~ms}$, with an echo time (TE) of $35 \mathrm{~ms}$. The field of view (FOV) was $230 \mathrm{~mm}$ with an acquisition matrix of $128 \mathrm{x}$ 128. Voxel size was $1.8 \mathrm{~mm} \times 1.8 \mathrm{~mm} \times 3.5 \mathrm{~mm}$. A T1-weighted structural image was obtained for each participant for use in image pre-processing.

\section{Scanning session}


A 5-minute eyes-closed resting state scan — the results of which are not reported here-was followed by the first touch-run, as described above. VAS ratings were not obtained during the scanning session to avoid engaging cognitive evaluative processes. Participants were not blindfolded and instructed to keep their eyes open, but all participants reported they were unable to see the brushes being administered. Participants were instructed to concentrate on how the stimuli felt throughout each touch-run. The first-touch run was followed by a 6-minute task involving self-administered touch, the results of which are not reported here. The second touch-run was then administered followed by a 6-minute structural brain scan and then the final touch-run. Each participant therefore experienced three $20 \mathrm{~s}$ blocks of stroking with each stimulus on both the left ventral forearm and glabrous skin of the fingers whilst in the scanner.

\section{Post scanning}

Following the scanning session, touch ratings were obtained as described previously. Due to the double-blind nature of this study, all participants were given a protein-rich meal of their choice at the end of the experimental session to replete endogenous tryptophan levels. This was followed by de-briefing the participant and checking for any residual tryptophan depletion effects before allowing them to return home.

\section{Data Analysis}

VAS \& plasma tryptophan analysis

VAS, POMS, FCPS and total plasma tryptophan data were analysed with SPSS version 22 (IBM Corp), using the multivariate approach to repeated-measures modelling (Rencher \& Christensen, 2012). Significant interaction effects were followed up using simple main effects and pairwise comparisons with Sidak correction (denoted in text as $p_{\mathrm{S}}$ ). Model assumptions were verified using model residual plots combined with the Shapiro-Wilk test of normality. 
Homogeneity of the covariance matrices between groups was verified by the use of Box's test. $F$ approximations to Pillai's trace are reported.

No significant difference between treatment groups was identified for VAS ratings obtained during screening. Additionally, no significant effect of treatment group was identified between VAS ratings obtained during screening and immediately post scanning. For these reasons, data obtained immediately post scanning were analysed alone to simplify incorporation of the between-subject factor of treatment group (TRP+/TRP-) in the analysis model, as well as the two within-subject factors of texture (soft/medium/coarse), and location (forearm/fingers). Examination of model residuals showed a departure from normality, so a square root transformation was used.

POMS, FCPS and total plasma tryptophan data were analysed separately. All models consisted of a within-subject factor of time (baseline, +4 hours after amino acid consumption) and a between-subject factor of treatment (TRP+/TRP-).

\section{Imaging Data Analysis}

Imaging data were analysed using MATLAB (The MathWorks, Inc.), Statistical Parametric Mapping (SPM) version $12 \mathrm{~b}$ and the Sandwich Estimator (SwE) SPM toolbox. Functional images were re-aligned to the first volume. The structural image was then coregistered to the mean functional image and segmented into its constituent tissue classes. The transformation to the standard Montreal Neurological Institute (MNI) space calculated from the segmentation procedure was then applied to the functional volumes before smoothing using a Gaussian kernel full width half maximum (FWHM) of $5.4 \times 5.4 \times 10.5$. As an additional motion correction step, the Artefact Detection Toolbox (ART, http://www.nitrc.org/projects/artifact_detect/) was used to identify outlying volumes based on a volume-to-volume shift of $>1 \mathrm{~mm}$ and a volume-to-volume change in mean signal intensity 
$>3$ standard deviations. If more than $15 \%$ of volumes per touch-run were identified as outliers, this run was excluded from the analysis. Only one run for one participant was excluded for this reason. For the remaining touch-runs a separate regressor for each outlying volume was included in the first level design matrix in order to 'censor' the effectors of motion from the parameter estimates (Power et al., 2012; Siegel et al., 2014). For the subject-level analysis, the parameter estimate for the preceding rest block was subtracted from the parameter estimate during the stimulation block. A $128 \mathrm{~s}$ high pass filter was used to account for low-frequency signal drift. The parameter estimates from each participant for each condition were then averaged across runs to take through to group-level modelling.

The group-level model had a single between subject factor of treatment group (TRP+/TRP-), and two within subject factors of location (forearm/fingers) and texture (soft/medium/coarse). In order to accommodate the repeated measurements at the group level we made use of the sandwich estimator toolbox (Guillaume et al., 2014), allowing us to fit a single marginal model with an unconstrained covariance structure at every voxel. Activations with False Discovery Rate (FDR) corrected $p$-values $\leq 0.05$ and cluster size $\geq 5$ voxels are reported for all contrasts. To investigate significant interactions, follow-up analysis involved small volume correction using the activation map for the interaction with threshold FDR $\leq$ 0.05. This allowed identification of significant effects within interactions.

From previous brain imaging studies of CT-targeted touch (Craig, 2002; Olausson et al., 2002; Lindgren et al., 2012; McGlone et al., 2012; Gordon et al., 2013), a single a-priori region of interest mask was used for all contrasts and interactions at the group-level. The mask consisted of orbitofrontal cortex (OFC), defined using the automated anatomical labelling (AAL) atlas (Tzourio-Mazoyer et al., 2002), postcentral gyrus, insula, anterior cingulate and amygdala, from the WFU PickAtlas (Lancaster et al., 1997, 2000; Maldjian et al., 2003, 2004), 
plus secondary somatosensory cortex (SII), defined by parietal operculum (OP) regions 1-4 from the Anatomy Toolbox version 1.8 (Eickhoff et al., 2005, 2006a, 2006b).

\section{Results}

Treatment groups (TRP+/TRP-) were comparable in terms of age $\left(\mathrm{t}_{21.46}=1.21, p=0.24\right)$ and IQ $\left(\mathrm{t}_{28}=0.74, p=0.46\right)($ Table 1$)$.

\section{Total plasma tryptophan levels}

Analysis of total plasma tryptophan concentration at baseline and 4 hours after drink consumption between TRP+ and TRP- treatment groups (see Table 1), revealed a significant interaction of treatment with time $\left(\mathrm{F}_{1,28}=353.148, p<0.001, \eta_{p}{ }^{2}=0.927\right.$, power $\left.=1.000\right)$. Analysis of simple effects identified a significant decrease in total plasma tryptophan concentrations 4 hours after drink administration in the TRP-group $\left(\mathrm{F}_{1,28}=72.211, p<0.001\right.$, $\eta_{p}{ }^{2}=0.721$, power $\left.=1.000\right)$ and a significant increase in the TRP + group $\left(\mathrm{F}_{1,28}=316.252, p<\right.$ $0.001, \eta_{p}{ }^{2}=0.919$, power $\left.=1.000\right)$. Plasma tryptophan concentrations of the TRP- group decreased by $74 \pm 1.3 \%$ (mean \pm S.E.) and increased $284 \pm 17.6 \%$ in the TRP + group. Average total plasma tryptophan concentrations reported for the current study before and after consumption of the amino acid drinks were comparable to those reported in previously published studies using ATD (Evers et al., 2006; Roiser et al., 2008; Bilderbeck et al., 2011).

\section{Self-reported mood}

There was no significant change in mood from baseline to 4 hours post amino acid consumption (POMS total mood disturbance (TMS): $\mathrm{F}_{1,28}=3.067, p=0.091, \eta_{p}{ }^{2}=0.099$, power $=0.394$. FCPS: $\mathrm{F}_{1,28}=2.514, p=0.124, \eta_{p}{ }^{2}=0.082$, power $\left.=0.334\right)$ and no significant interaction with treatment group (TRP+/TRP-) (POMS TMS: $\mathrm{F}_{1,28}=0.016, p=0.900, \eta_{p}^{2}=$ 
0.001 , power $=0.052$. FCPS: $\mathrm{F}_{1,28}=0.187, p=0.669, \eta_{p}{ }^{2}=0.007$, power $\left.=0.070\right)$. These data are presented in Table 1.

\section{Touch Ratings}

No effect of ATD on touch ratings was identified. A significant interaction of location with texture was identified $\left(\mathrm{F}_{2,27}=12.555, p<0.001, \eta_{p}{ }^{2}=0.482\right.$, power $\left.=0.992\right)$. At both locations, the soft brush was rated most pleasant, the coarse brush least pleasant and the medium brush significantly less pleasant than the soft brush (forearm: $\mathrm{t}_{28}=7.510, p_{S}<0.001$; fingers: $\mathrm{t}_{28}=$ 4.613, $p_{S}<0.001$ ) and significantly more pleasant than the coarse brush (forearm: $t_{28}=6.223$, $p_{S}<0.001$; fingers: $\left.t_{28}=7.674, p_{S}<0.001\right)$. The soft brush was significantly more pleasant when applied to the forearm than the fingers $\left(\mathrm{F}_{1,29}=5.854, p=0.022, \eta_{p}^{2}=0.168\right.$, power $=$ 0.648). For the medium brush, application to the fingers was significantly more pleasant than the forearm $\left(\mathrm{F}_{1,29}=12.949, p=0.001, \eta_{p}{ }^{2}=0.309\right.$, power $\left.=0.935\right)$. For the coarse brush, application to the forearm was significantly more unpleasant than to the fingers $\left(\mathrm{F}_{1,29}=5.838\right.$, $p=0.022, \eta_{p}^{2}=0.168$, power $\left.=0.646\right)$. The main effect of texture was also significant $\left(\mathrm{F}_{2,27}\right.$ $=44.626, p<0.001, \eta_{p}{ }^{2}=0.768$, power $\left.=1.000\right)$. Graphical representation of this data is presented in Figure 1.

\section{fMRI data analysis}

Effect of treatment and touch location on BOLD response

ATD reduced the differential response of the ipsilateral Inferior Frontal Gyrus (IFG) region of lateral OFC (Brodmann Area (BA)47) to CT-targeted vs non-targeted touch. As shown in Figure 2 and Table 2, a significant interaction of treatment (TRP+/TRP-) with location (forearm/fingers) was identified in the ipsilateral IFG region of lateral OFC (BA47). Followup contrasts revealed CT-targeted forearm touch produced a significantly greater BOLD 
response than $\mathrm{CT}$ non-targeted touch to the fingers in the TRP+ condition. This differential response to CT activating vs non-activating touch was not present in the TRP-group, where the BOLD response to touch to the forearm and fingers was comparable. The BOLD response to touch to the forearm and fingers did not differ significantly between treatment groups.

Pearson's correlations were used to determine whether touch ratings significantly correlated with BOLD response of the peak voxel (MNI -37 25 -18). No significant correlations were identified between hedonic ratings of pleasantness and BOLD response.

\section{Effect of treatment, stimulus location and texture on BOLD response}

ATD altered contralateral SI response to discriminatory touch. A significant interaction of treatment group (TRP+/TRP-) with stimulus texture (soft/medium/coarse) and touch location (forearm/fingers) was identified in contralateral SI, as shown in Figure 3 and Table 2. Follow up contrasts to further investigate this interaction revealed touch to the fingers, where $A \beta$ innervation density is high and CTs are absent, produced a differential contralateral SI response to brush texture in the TRP+ group, with the coarse brush producing significantly greater activation than the soft brush. Contralateral SI response to touch to the fingers in the TRPgroup did not show this differential response to texture, in fact, SI response to all three brush textures was comparable. This effect was not seen following touch to the forearm where A $\beta$ innervation density is much lower. As expected, overall SI response to touch to the fingers was significantly greater than touch to the forearm. Pearson's correlations of hedonic ratings of touch with BOLD response of the peak voxel (MNI: 44 -27 53) were not significant.

\section{Effect of stimulus location and texture on BOLD response}

A significant interaction of location (forearm/fingers) with texture (soft/medium/coarse) was identified in ipsilateral SI and contralateral OP4 of SII, as presented in Table 2. Follow up 
contrasts to investigate the effect of texture at each location separately revealed no significant differences between textures for touch to the forearm or fingers in SI or SII after FDR correction. In ipsilateral SI, the effect of location for each texture revealed a significant difference between the forearm and fingers, consistent with the main effect of location identified in this region (see Table 3). In SII no significant difference between the forearm and fingers was identified for any of the textures after FDR correction.

Comparison of BOLD response to CT-targeted vs non-targeted touch

Brain regions with significantly different BOLD responses to CT-targeted touch to the forearm compared to touch to the fingers where no $\mathrm{CT}$ innervation is present are presented in Table 3 and Figure 4. Consistent with previous studies, CT-targeted forearm touch produced significantly greater BOLD response in limbic regions (anterior cingulate and contralateral posterior insula) than $\mathrm{CT}$ non-targeted touch to the fingers. Touch to the fingers produced significantly greater BOLD response in somatosensory regions (bilateral SI and contralateral SII (OP1)) and mid-insula than touch to the forearm.

\section{Effect of touch to the forearm and fingers compared to rest}

Contrast of forearm touch compared to rest identified significant activation of the ipsilateral IFG region of lateral OFC (BA47), bilateral SII and contralateral posterior insula and SI. Touch to the fingers compared to rest resulted in significant activation of bilateral SI and the IFG region of lateral OFC (BA47) and ipsilateral mid-insula and SII (Table 4).

\section{Discussion}

Overview of results 
This study replicated previous findings that CT-targeted touch (forearm - finger) preferentially activates affective processing regions; OFC, anterior cingulate and posterior insula (Olausson et al., 2002, 2008b; Hua et al., 2008; Lindgren et al., 2012; McGlone et al., 2012; Gordon et al., 2013; Voos et al., 2013; Kaiser et al., 2015), whereas A $\beta$ mediated touch (finger - forearm) primarily activated somatosensory processing regions; SI and SII. Tryptophan depletion reduced circulating tryptophan concentrations by $74 \%$ and modulated central responses to CT-targeted and purely discriminatory touch without modifying hedonic ratings of pleasantness. In keeping with previous studies in non-vulnerable healthy volunteers, tryptophan depletion did not affect self-rated mood (Roiser et al., 2008; Bilderbeck et al., 2011).

Serotonin modulates the differential OFC response to CT activating vs non-activating touch

Irrespective of brush texture, stroking of the forearm evoked greater subjective responses than when applied to the finger as previously reported and in keeping with the CT-affective touch hypothesis (Vallbo et al., 1999; Olausson et al., 2008b; Morrison et al., 2010; McGlone et al., 2014). However, stimulus texture did not affect the central responses to CT activating touch to the forearm. This agrees with Morrison et al. (2011), who also found no significant difference in brain regions associated with CT encoding when comparing responses between a soft and stiff brush. In contrast to the current study, Morrison et al. (2011), reported a significant differential SI response for soft compared to stiff brush application to the forearm, but both studies support the hypothesis that CTs do not specifically encode pleasant touch per se, rather, they encode the stimulus velocity (Loken et al., 2009) and temperature (Ackerley et al., 2014) associated with interpersonal social interactions. Thus the main role of CTs may be to signal that a potentially affiliative gesture (stroking) has occurred which is independent of other dimensions such as the condition of the skin or texture of the stimulus. 
A key region of the brain in which this signalling is modulated by serotonin was identified in the IFG region of lateral OFC (BA47) where touch to the forearm elicited BOLD responses regardless of stimulus texture, with no responses to non-CT directed stimulation of the finger. This selectivity of response to CT activating touch replicates a previously reported positron emission tomography (PET) investigation of healthy individuals where the IFG region of lateral OFC (BA47) was significantly more activated by CT-targeted touch to the forearm compared to CT non-targeted touch to the palm (McGlone et al., 2012). After tryptophan depletion both forearm and finger stimuli elicited BOLD responses suggesting both classes of stimuli can engage the IFG region of lateral OFC (BA47), as shown by activation of this region by finger and forearm stimulation (Table 4). Serotonin may thus play an important role in tuning the IFG region of lateral OFC (BA47) responses to CT touch by inhibiting non-CT input. That the modulation occurs centrally and possibly locally, is suggested by the absence of tryptophan depletion effects in other regions that respond to CT-targeted stimulation such as posterior insula and anterior cingulate. In keeping with Deakin and Graeff(1991), it is possible that CT stimulation may activate serotonin projections which suppress the non-CT input into lateral OFC revealed by tryptophan depletion.

A number of studies suggest that the IFG region of lateral OFC (BA47) is an important region for processing socially relevant and affective stimuli such as images of face emotions (Goulden et al., 2012) or social inclusion/exclusion (Elliott et al., 2012), and affective speech prosody (Wildgruber et al., 2004). Furthermore, four studies report tryptophan depletion modulation of fMRI activations by affective faces, images or words in the IFG region of lateral OFC (BA47) in healthy volunteers (Fusar-Poli et al., 2007; Williams et al., 2007; Wang et al., 2009; Daly et al., 2010). Recent evidence suggests neuronal populations in medial and lateral OFC represent a supramodal continuum of valence of stimuli from unpleasant to pleasant, irrespective of stimulus modality (Chikazoe et al., 2014). One possibility is that the IFG region 
of lateral OFC (BA47) contributes to a social valence or affiliative system based on the integration of a variety of sensory modalities that influence social decision-making (Bzdok et al., 2012) such as visual (e.g. face emotion) (Goulden et al., 2012), auditory (emotional prosody) (Wildgruber et al., 2004) and, as our results suggest, tactile information. This study indicates serotonin maintains a bias for the IFG region of lateral OFC (BA47) to respond to socially relevant CTs. This could be one component mediating the prosocial functions of serotonin seen in the subjective effects of MDMA (Bedi et al., 2009; Wardle et al., 2014) and in the effects of citalopram and tryptophan depletion in experimental studies of social cooperation and moral behaviour (Wood et al., 2006; Crockett et al., 2010; Bilderbeck et al., 2011, 2013; Siegel \& Crockett, 2013).

Preferential somatosensory responses to finger stroking; modulation by tryptophan depletion

For control participants, stroking stimulation of the finger evoked greater responses for all textures (coarse $>$ medium $>$ soft) in contralateral SI than CT directed forearm stimuli which evoked no measurable responses in this region. The fingers are adapted for fine discriminatory touch perception with a dense innervation of $\mathrm{A} \beta$ afferents, a large representation in SI and much finer two-point discrimination compared to the forearm (v. Békésy, 1957; Verrillo \& Chamberlain, 1972; Johansson \& Vallbo, 1979). The results of the present study confirm this, with significantly greater SI activation following finger compared to forearm stimulation.

The graded BOLD response to coarseness was absent in the tryptophan depleted group, implicating a possible role for serotonin in texture discrimination. Centrally, previous investigations in the rat have found serotonin to modulate the spontaneous compared to stimulus specific responses of $A \beta$ mechanosensitive neuronal pathways at the level of both the thalamus (Starr et al., 2008) and SI (Waterhouse et al., 1986). Serotonin has been found to predominantly depolarise interneurons in layers II and III of rat SI, depolarise the majority of 
layer I neurons projecting to layers II and III and hyperpolarise the majority of layer I neurons whose axons remain in layer I (Foehring et al., 2002). Serotonin therefore alters neuronal firing patterns in SI and has been suggested to alter the temporal components of sensory neuron responses which allow the encoding of different surface texture properties (Hurley et al., 2004). By reducing central serotonin through ATD, SI neuronal responses, including their temporal components, may be altered to the extent that responses to different surface textures becomes less specific, as seen in the SI response reported in the current study.

The differential SI response seen in the non-depleted group could additionally reflect a peripheral mechanism altered by tryptophan depletion. The coarse features of a texture, such as braille dots and gratings, are encoded peripherally by the spatially modulated neural signal of slowly adapting type 1 (SA1) afferents, which densely innervate the fingers (Yoshioka et al., 2001). Weber et al. (2013) demonstrated rapidly adapting (RA) and Pacinian (PC) afferents are required to encode texture-specific vibrations propagated by the stimulus moving over the skin, allowing finer surface properties to be encoded. SA1 afferents form Merkel cell-neurite complexes in the basal layer of the epidermis (Haeberle \& Lumpkin, 2008), which mediate SA1 responses (Nakatani et al., 2014). Immunohistochemistry has identified serotonin, 5-HT $1 \mathrm{~A}$ and 5-HT $1 \mathrm{~B}$ receptors and the 5-HT transporter in Merkel cell-neurite complexes (English et al., 1992; Tachibana et al., 2005). 5- $\mathrm{HT}_{2}$ and 5-HT 3 antagonists alter SA1 response to mechanical stimulation (He et al., 2003). Additionally, 5- $\mathrm{HT}_{2 \mathrm{~A}}$ receptors have been identified on Pacinian Corpuscles (Carlton \& Coggeshall, 1997). Further investigation into the modulatory role of serotonin in $\mathrm{A} \beta$ mediated mechanosensation is required, including whether this is a purely central process, or whether peripheral mechanisms are also involved.

\section{Wider implications and conclusions}


Deakin and Graeff (1991) hypothesised that touch-induced activation of serotonin pathways might contribute to the protective effects of social support against depression. The discovery of the CT system and its potential role in affective touch raised the possibility that CT simulation may activate serotonin pathways which in turn could mediate the pleasantness of affective touch. However, in this study we did not find that tryptophan depletion reduced subjective pleasantness ratings to CT-targeted touch but that serotonin promotes a bias to CT responsiveness in the IFG region of lateral OFC (BA47), but in no other brain regions previously implicated in $\mathrm{CT}$ touch responses, indicating a modulatory rather than a mediating role of serotonin in processing CT-targeted touch. Nevertheless, it remains possible that CT afferent activity itself evokes the tonic serotonin release which supresses responses to non-CT stimuli in OFC and which could modulate other systems to promote resilience to stress and depression. Studies of the CT system in patients with depression and the modulatory influence of serotonin, especially in the IFG region of lateral OFC (BA47), would seem worthwhile.

A lack of response to CT stimulation in the IFG region of lateral OFC (BA47) has been reported in spectrum disorder (ASD) (Kaiser et al., 2015). ASD involves impaired social communication (Pelphrey et al., 2011) and is associated with abnormal serotonin function (Chugani et al., 1999; Azmitia et al., 2011; Oblak et al., 2013) and with hyper- and hypo-reactivity to sensory input specified in the Diagnostic and Statistical Manual of Mental Disorders (DSM-5) of the American Psychiatric Association (2013). Kaiser et al., (2015) found that the IFG region of lateral OFC responses (BA47) to CT-targeted compared to non-targeted touch seen in control participants, were absent in an ASD group. While this is similar to the effect of tryptophan depletion in our study, it is not clear whether the ASD effect is due to disinhibition of non-CT targeted touch as in our tryptophan depletion finding rather than an absence of CT responses. 
It is clearly important to determine whether the peripheral CT system is functionally intact in ASD and whether abnormal CNS representation could be modulated by serotonin.

Limitations in this study include the small sample size, between-subject design and femaleonly participants. It is also worth noting that other neurotransmitters have been implicated in CT-targeted touch and social behaviour such as oxytocin (Ellingsen et al., 2014; Scheele et al., 2014) and opioids (Case et al., 2016). Nevertheless, the results provide preliminary evidence of a modulatory role of serotonin in the differentiation between socially relevant CT activating touch and purely discriminatory touch responses. This provides one mechanism by which social deficits and altered touch responses may be observed in psychiatric disorders, such as depression and autism, in which dysfunction of the serotonin system has been implicated. Further investigation into the role of social touch in a range of psychiatric disorders will allow us to expand our knowledge of the neurobiological mechanisms underlying these disorders.

\section{Acknowledgements}

This work was supported by a doctoral Collaborative Award in Science and Engineering (CASE) grant from the Biotechnology and Biological Sciences Research Council (BBSRC) and Unilever (FA01181 to P.D.T.) and by a University of Manchester Translational Imaging Unit grant (MRH035 to P.D.T). F.M., S.C.W. \& P.D.T. currently receive funding from a Leverhulme Trust grant (RPG-2013-058). Funding sources were not involved in study design, collection, analysis or interpretation of data, in writing this report, or deciding to submit this article for publication.

\section{Abbreviations}

ASD, Autism Spectrum Disorder; ATD, Acute Tryptophan Depletion; BA, Brodmann Area; BOLD, Blood Oxygenation Level Dependent; CNS, Central Nervous System; CTs, C-tactile 
afferents; FCPS, Fawcett-Clark Pleasure Scale; FDR, False Discovery Rate; IFG, Inferior Frontal Gyrus; IQ, Intelligence Quotient; OFC, Orbitofrontal Cortex; OP, Parietal Operculum; POMS, Profile Of Mood States; SA1, Slowly Adapting type 1; SI, Primary Somatosensory Cortex; SII, Secondary Somatosensory Cortex; VAS, Visual Analogue Scale

\section{References}

Ackerley, R., Wasling, H.B., Liljencrantz, J., Olausson, H., Johnson, R.D., \& Wessberg, J. (2014) Human C-tactile afferents are tuned to the temperature of a skin-stroking caress. J. Neurosci., 34, 2879-2883.

American Psychiatric Association, A.P.A. (2013) Diagnostic and Statistical Manual of Mental Disorders, Fifth Edition. American Psychiatric Association, Arlington, VA.

Ammons, R.B. \& Ammons, C.H. (1960) The Quick Test (QT): provisional manual. Psychol Reports, 11, 111-161.

Azmitia, E.C., Singh, J.S., \& Whitaker-azmitia, P.M. (2011) Increased serotonin axons (immunoreactive to 5-HT transporter) in postmortem brains from young autism donors. Neuropharmacology, 60, 1347-1354.

Bedi, G., Phan, K.L., Angstadt, M., \& de Wit, H. (2009) Effects of MDMA on sociability and neural response to social threat and social reward. Psychopharmacology (Berl)., 207, $73-83$.

Bell, C.J., Hood, S.D., \& Nutt, D.J. (2005) Acute tryptophan depletion. Part II: clinical effects and implications. Aust N Z J Psychiatry, 39, 565-574.

Benkelfat, C., Ellenbogen, M.A., Dean, P., Palmour, R.M., \& Young, S.N. (1994) Moodlowering effect of tryptophan depletion. Enhanced susceptibility in young men at genetic risk for major affective disorders. Arch Gen Psychiatry, 51, 687-697.

Bilderbeck, A.C., McCabe, C., Wakeley, J., McGlone, F., Harris, T., Cowen, P.J., \& Rogers, R.D. (2011) Serotonergic activity influences the cognitive appraisal of close intimate relationships in healthy adults. Biol. Psychiatry, 69, 720-725.

Bilderbeck, A.C., Wakeley, J., Godlewska, B.R., McGlone, F., Harris, T., Cowen, P.J., \& Rogers, R.D. (2013) Preliminary evidence that sub-chronic citalopram triggers the reevaluation of value in intimate partnerships. Soc. Cogn. Affect. Neurosci., 9, 1419-1425.

Bjornsdotter, M., Loken, L., Olausson, H., Vallbo, A., \& Wessberg, J. (2009) Somatotopic organization of gentle touch processing in the posterior insular cortex. J Neurosci, 29, 9314-9320.

Brown, G.W., Craig, T.K., Harris, T.O., Handley, R. V, \& Harvey, A.L. (2007) Development of a retrospective interview measure of parental maltreatment using the Childhood Experience of Care and Abuse (CECA) instrument -- A life-course study of adult chronic depression - 1. J. Affect. Disord., 103, 205-215.

Burleson, M.H. \& Davis, M.C. (2014) Social Touch and Resilience. In Kent, M. et al. (eds), 
The Resilience Handbook: Approaches to Stress and Trauma. Routledge, Hove, pp. 131143.

Bystrova, K., Ivanova, V., Edhborg, M., Matthiesen, A.S., Ransjö-Arvidson, A.B., Mukhamedrakhimov, R., Uvnäs-Moberg, K., \& Widström, A.M. (2009) Early contact versus separation: Effects on mother-infant interaction one year later. Birth, 36, 97-109.

Bzdok, D., Langner, R., Hoffstaedter, F., Turetsky, B.I., Zilles, K., \& Eickhoff, S.B. (2012) The modular neuroarchitecture of social judgments on faces. Cereb. Cortex, 22, 951961.

Carlton, S.M. \& Coggeshall, R.E. (1997) Immunohistochemical localization of 5-HT2A receptors in peripheral sensory axons in rat glabrous skin. Brain Res., 763, 271-275.

Case, L.K., Ceko, M., Gracely, J.L., Richards, E.A., Olausson, H., \& Bushnell, M.C. (2016) Touch perception altered by chronic pain and by opioid blockade. eNeuro, 3, 1-10.

Chikazoe, J., Lee, D.H., Kriegeskorte, N., \& Anderson, A.K. (2014) Population coding of affect across stimuli, modalities and individuals. Nat. Neurosci., 17, 1114-1122.

Chugani, D.C., Muzik, O., Behen, M., Rothermel, R., Janisse, J.J., Lee, J., \& Chugani, H.T. (1999) Developmental changes in brain serotonin synthesis capacity in autistic and nonautistic children. Ann. Neurol., 45, 287-295.

Craig, A.D. (2002) How do you feel? Interoception: the sense of the physiological condition of the body. Nat Rev Neurosci, 3, 655-666.

Crockett, M.J., Clark, L., Hauser, M.D., \& Robbins, T.W. (2010) Serotonin selectively influences moral judgment and behavior through effects on harm aversion. Proc. Natl. Acad. Sci. U. S. A., 107, 17433-17438.

Daly, E., Deeley, Q., Hallahan, B., Craig, M., Brammer, M., Lamar, M., Cleare, A., Giampietro, V., Ecker, C., Page, L., Toal, F., Phillips, M.L., Surguladze, S., \& Murphy, D.G. (2010) Effects of acute tryptophan depletion on neural processing of facial expressions of emotion in humans. Psychopharmacology, 210, 499-510.

Daly, E., Ecker, C., Hallahan, B., Deeley, Q., Craig, M., Murphy, C., Johnston, P., Gillan, N., Gudbrandsen, M., Brammer, M., Giampietro, V., Lamar, M., Page, L., Toal, F., Schmitz, N., Cleare, A., Robertson, D., Rubia, K., \& Murphy, D.G.M. (2014) Response inhibition and serotonin in autism : a functional MRI study using acute tryptophan depletion. Brain, 137, 2600-2610.

Daly, E.M., Deeley, Q., Ecker, C., Craig, M., Hallahan, B., Murphy, C., Johnston, P., Spain, D., Gillan, N., Brammer, M., Giampietro, V., Lamar, M., Page, L., Toal, F., Cleare, A., Surguladze, S., \& Murphy, D.G.M. (2012) Serotonin and the neural processing of facial emotions in adults with autism: an fMRI study using acute tryptophan depletion. Arch. Gen. Psychiatry, 69, 1003-1013.

Deakin, J.F.W. (1996) 5HT antidepressants drugs and the psychosocial origins of depression. J. Psychopharmacol., 10, 31-38.

Deakin, J.F.W. \& Graeff, F.G. (1991) 5-HT and mechanisms of defence. J Psychopharmacol, 5, 305-315.

Delgado, P.L., Charney, D.S., Price, L.H., Aghajanian, G.K., Landis, H., \& Heninger, G.R. (1990) Serotonin function and the mechanism of antidepressant action. Reversal of antidepressant-induced remission by rapid depletion of plasma tryptophan. Arch. Gen. 
Psychiatry, 47, 411-418.

Derogatis, L.R. (1993) BSI: Brief Symptom Inventory: Administration, Scoring, and Procedures Manual. National Computer Systems Pearson, Inc., Minneapolis (MN).

Eickhoff, S.B., Amunts, K., Mohlberg, H., \& Zilles, K. (2006a) The human parietal operculum. II. Stereotaxic maps and correlation with functional imaging results. Cereb Cortex, 16, 268-279.

Eickhoff, S.B., Schleicher, A., Zilles, K., \& Amunts, K. (2006b) The human parietal operculum. I. Cytoarchitectonic mapping of subdivisions. Cereb. Cortex, 16, 254-267.

Eickhoff, S.B., Stephan, K.E., Mohlberg, H., Grefkes, C., Fink, G.R., Amunts, K., \& Zilles, K. (2005) A new SPM toolbox for combining probabilistic cytoarchitectonic maps and functional imaging data. Neuroimage, 25, 1325-1335.

Ellingsen, D.M., Wessberg, J., Chelnokova, O., Olausson, H., Laeng, B., \& Leknes, S. (2014) In touch with your emotions: Oxytocin and touch change social impressions while others' facial expressions can alter touch. Psychoneuroendocrinology, 39, 11-20.

Elliott, R., Lythe, K., Lee, R., McKie, S., Juhasz, G., Thomas, E.J., Downey, D., Deakin, J.F.W., \& Anderson, I.M. (2012) Reduced Medial Prefrontal Responses to Social Interaction Images in Remitted Depression. Arch. Gen. Psychiatry, 69, 37-45.

English, K.B., Wang, Z.Z., Stayner, N., Stensaas, L.J., Martin, H., \& Tuckett, R.P. (1992) Serotonin-like immunoreactivity in Merkel cells and their afferent neurons in touch domes from the hairy skin of rats. Anat. Rec., 232, 112-120.

Essick, G.K., McGlone, F., Dancer, C., Fabricant, D., Ragin, Y., Phillips, N., Jones, T., \& Guest, S. (2010) Quantitative assessment of pleasant touch. Neurosci Biobehav Rev, 34, 192-203.

Evers, E. a T., Sambeth, a, Ramaekers, J.G., Riedel, W.J., \& van der Veen, F.M. (2010) The effects of acute tryptophan depletion on brain activation during cognition and emotional processing in healthy volunteers. Curr. Pharm. Des., 16, 1998-2011.

Evers, E. a T., Van Der Veen, F.M., Jolles, J., Deutz, N.E.P., \& Schmitt, J. a J. (2006) Acute tryptophan depletion improves performance and modulates the bold response during a stroop task in healthy females. Neuroimage, 32, 248-255.

Fawcett, J., Clark, D.C., Scheftner, W.A., \& Gibbons, R.D. (1983) Assessing anhedonia in psychiatric patients: The pleasure scale. Arch Gen Psychiatry, 40, 79-84.

Feldman, R., Rosenthal, Z., \& Eidelman, A.I. (2014) Maternal-preterm skin-to-skin contact enhances child physiologic organization and cognitive control across the first 10 years of life. Biol. Psychiatry, 75, 56-64.

Feldman, R., Singer, M., \& Zagoory, O. (2010) Touch attenuates infants' physiological reactivity to stress. Dev. Sci., 13, 271-278.

Field, T. (2001) Massage Therapy Facilitates Weight Gain in Preterm Infants. Curr. Dir. Psychol. Sci., 10, 51-54.

Field, T. (2010) Touch for socioemotional and physical well-being: A review. Dev. Rev., 30, $367-383$.

First, M.B., Spitzer, R.L., Gibbon, M., \& Williams, J.B.W. (2002) Structured Clinical Interview for DSM-IV-TR Axis I Disorders - Non-Patient Edition (SCID-I/NP, 11/2002 
Revision). Biometric Research Department, New York State Psychiatric Institute, New York (NY).

Foehring, R.C., van Brederode, J.F.M., Kinney, G.A., \& Spain, W.J. (2002) Serotonergic Modulation of Supragranular Neurons in Rat Sensorimotor Cortex. J. Neurosci., 22, 8238-8250.

Fusar-Poli, P., Allen, P., Lee, F., Surguladze, S., Tunstall, N., Fu, C.H.Y., Brammer, M.J., Cleare, A.J., \& McGuire, P.K. (2007) Modulation of neural response to happy and sad faces by acute tryptophan depletion. Psychopharmacology (Berl)., 193, 31-44.

Gordon, I., Voos, A.C., Bennett, R.H., Bolling, D.Z., Pelphrey, K.A., \& Kaiser, M.D. (2013) Brain mechanisms for processing affective touch. Hum Brain Mapp, 34, 914-922.

Goulden, N., McKie, S., Thomas, E.J., Downey, D., Juhasz, G., Williams, S.R., Rowe, J.B., Deakin, J.F.W., Anderson, I.M., \& Elliott, R. (2012) Reversed frontotemporal connectivity during emotional face processing in remitted depression. Biol. Psychiatry, 72, 604-611.

Guillaume, B., Hua, X., Thompson, P.M., Waldorp, L., \& Nichols, T.E. (2014) Fast and accurate modelling of longitudinal and repeated measures neuroimaging data. Neuroimage, 94, 287-302.

Haeberle, H. \& Lumpkin, E. a. (2008) Merkel Cells in Somatosensation. Chemosens. Percept., 1, 110-118.

Hamet, P. \& Tremblay, J. (2005) Genetics and genomics of depression. Metabolism., 54, 10 15.

He, L., Tuckett, R.P., \& English, K.B. (2003) 5-HT2 and 3 receptor antagonists suppress the response of rat type I slowly adapting mechanoreceptor: An in vitro study. Brain Res., 969, 230-236.

Hood, S.D., Bell, C.J., \& Nutt, D.J. (2005) Acute tryptophan depletion. Part I: rationale and methodology. Aust. N. Z. J. Psychiatry, 39, 558-564.

Hua, Q.P., Zeng, X.Z., Liu, J.Y., Wang, J.Y., Guo, J.Y., \& Luo, F. (2008) Dynamic changes in brain activations and functional connectivity during affectively different tactile stimuli. Cell. Mol. Neurobiol., 28, 57-70.

Hurley, L.M., Devilbiss, D.M., \& Waterhouse, B.D. (2004) A matter of focus: Monoaminergic modulation of stimulus coding in mammalian sensory networks. Curr. Opin. Neurobiol., 14, 488-495.

Johansson, R.S. \& Vallbo, A.B. (1979) Tactile Sensibility in the Human Hand: Relative and Absolute Densities of Four Types of Mechanoreceptive Units in Glabrous Skin. $J$. Physiol., 286, 283-300.

Kaiser, M.D., Yang, D.Y.-J., Voos, A.C., Bennett, R.H., Gordon, I., Pretzsch, C., Beam, D., Keifer, C., Eilbott, J., McGlone, F., \& Pelphrey, K.A. (2016) Brain Mechanisms for Processing Affective (and Nonaffective) Touch Are Atypical in Autism. Cereb. Cortex, 26, 2705-2714.

Kiser, D., SteemerS, B., Branchi, I., \& Homberg, J.R. (2012) The reciprocal interaction between serotonin and social behaviour. Neurosci. Biobehav. Rev., 36, 786-798.

Klein, H., Elifson, K.W., \& Sterk, C.E. (2009) Young Adult Ecstasy Users' Enhancement of 
the Effects of Their Ecstasy Use. J Psychoact. Drugs, 41, 113-120.

Lancaster, J., Summerln, J., Rainey, L., Freitas, C., \& Fox, P. (1997) The Talairach Daemon, a database server for Talairach Atlas Labels. Neuroimage, 5, S633.

Lancaster, J.L., Woldorff, M.G., Parsons, L.M., Liotti, M., Freitas, C.S., Rainey, L., Kochunov, P. V., Nickerson, D., Mikiten, S.A., \& Fox, P.T. (2000) Automated Talairach Atlas labels for functional brain mapping. Hum. Brain Mapp., 10, 120-131.

Lindgren, L., Westling, G., Brulin, C., Lehtipalo, S., Andersson, M., \& Nyberg, L. (2012) Pleasant human touch is represented in pregenual anterior cingulate cortex. Neuroimage, 59, 3427-3432.

Loken, L.S., Wessberg, J., Morrison, I., McGlone, F., \& Olausson, H. (2009) Coding of pleasant touch by unmyelinated afferents in humans. Nat. Neurosci., 12, 547-548.

Maldjian, J. a., Laurienti, P.J., Kraft, R. a., \& Burdette, J.H. (2003) An automated method for neuroanatomic and cytoarchitectonic atlas-based interrogation of fMRI data sets. Neuroimage, 19, 1233-1239.

Maldjian, J.A., Laurienti, P.J., \& Burdette, J.H. (2004) Precentral gyrus discrepancy in electronic versions of the Talairach atlas. Neuroimage, 21, 450-455.

McGlone, F., Olausson, H., Boyle, J.A., Jones-Gotman, M., Dancer, C., Guest, S., \& Essick, G. (2012) Touching and feeling: differences in pleasant touch processing between glabrous and hairy skin in humans. Eur J Neurosci, 35, 1782-1788.

McGlone, F., Vallbo, A.B., Olausson, H., Loken, L., \& Wessberg, J. (2007) Discriminative touch and emotional touch. Can. J. Exp. Psychol. Can. Psychol. expérimentale, 61, 173.

McGlone, F., Wessberg, J., \& Olausson, H. (2014) Discriminative and Affective Touch: Sensing and Feeling. Neuron, 82, 737-755.

McNair, D.M. \& Lorr, M. (1971) Manual for the Profile of Mood States (POMS). Educational and Industrial Testing Service, San Diego (CA).

Meaney, M.J. \& Szyf, M. (2005) Maternal care as a model for experience-dependent chromatin plasticity? Trends Neurosci., 28, 456-463.

Moore, E.R., Anderson, G.C., Bergman, N., \& Dowswell, T. (2012) Early skin-to-skin contact for mothers and their healthy newborn infants. Cochrane database Syst. Rev., $\mathbf{5}$, CD003519.

Morrison, I., Bjornsdotter, M., \& Olausson, H. (2011) Vicarious responses to social touch in posterior insular cortex are tuned to pleasant caressing speeds. J. Neurosci., 31, 95549562.

Morrison, I., Loken, L.S., \& Olausson, H. (2010) The skin as a social organ. Exp. Brain Res., 204, 305-314.

Morton, J. (2005) Ecstasy: pharmacology and neurotoxicity. Curr Opin Pharmacol, 5, 79-86.

Nakatani, M., Maksimovic, S., Baba, Y., \& Lumpkin, E. a. (2014) Mechanotransduction in epidermal Merkel cells. Pflügers Arch. - Eur. J. Physiol., 101-108.

Nishizawa, S., Benkelfat, C., Young, S.N., Leyton, M., Mzengeza, S., de Montigny, C., Blier, P., \& Diksic, M. (1997) Differences between males and females in rates of serotonin synthesis in human brain. Proc Natl Acad Sci U S A, 94, 5308-5313. 
Oblak, A., Gibbs, T.T., \& Blatt, G.J. (2013) Reduced serotonin receptor subtypes in a limbic and a neocortical region in autism. Autism Res., 6, 571-583.

Olausson, H., Cole, J., Rylander, K., McGlone, F., Lamarre, Y., Wallin, B.G., Kramer, H., Wessberg, J., Elam, M., Bushnell, M.C., \& Vallbo, A. (2008a) Functional role of unmyelinated tactile afferents in human hairy skin: sympathetic response and perceptual localization. Exp Brain Res, 184, 135-140.

Olausson, H., Lamarre, Y., Backlund, H., Morin, C., Starck, G., Ekholm, S., Strigo, I., \& Worsley, K. (2002) Unmyelinated tactile afferents signal touch and project to the insular cortex. Nat Neurosci, 5, 900-904.

Olausson, H.W., Cole, J., Vallbo, A., McGlone, F., Elam, M., Kramer, H.H., Rylander, K., Wessberg, J., \& Bushnell, M.C. (2008b) Unmyelinated tactile afferents have opposite effects on insular and somatosensory cortical processing. Neurosci Lett, 436, 128-132.

Pelphrey, K.A., Shultz, S., Hudac, C.M., \& Vander Wyk, B.C. (2011) Research review: Constraining heterogeneity: the social brain and its development in autism spectrum disorder. J. Child Psychol. Psychiatry., 52, 631-644.

Power, J.D., Barnes, K.A., Snyder, A.Z., Schlaggar, B.L., \& Petersen, S.E. (2012) Spurious but systematic correlations in functional connectivity MRI networks arise from subject motion. Neuroimage, 59, 2142-2154.

Psychology Software Tools, I. (2012) E-Prime. Retrieved from http://www.pstnet.com.

Rencher, A.C. \& Christensen, W.F. (2012) Methods of Multivariate Analysis, Third Edit. edn. Wiley-Blackwell, Hoboken, New Jersey.

Roiser, J.P., Levy, J., Fromm, S.J., Wang, H., Hasler, G., Sahakian, B.J., \& Drevets, W.C. (2008) The effect of acute tryptophan depletion on the neural correlates of emotional processing in healthy volunteers. Neuropsychopharmacology, 33, 1992-2006.

Scheele, D., Kendrick, K.M., Khouri, C., Kretzer, E., Schläpfer, T.E., Stoffel-Wagner, B., Güntürkün, O., Maier, W., \& Hurlemann, R. (2014) An oxytocin-induced facilitation of neural and emotional responses to social touch correlates inversely with autism traits. Neuropsychopharmacology, 39, 2078-2085.

Siegel, J.S., Power, J.D., Dubis, J.W., Vogel, A.C., Church, J. a, Schlaggar, B.L., \& Petersen, S.E. (2014) Statistical improvements in functional magnetic resonance imaging analyses produced by censoring high-motion data points. Hum. Brain Mapp., 35, 1981-1996.

Siegel, J.Z. \& Crockett, M.J. (2013) How serotonin shapes moral judgment and behavior. Ann. N. Y. Acad. Sci., 1299, 42-51.

Starr, M.A., Page, M.E., \& Waterhouse, B.D. (2008) MDMA (3,4methylenedioxymethamphetamine)-mediated distortion of somatosensory signal transmission and neurotransmitter efflux in the ventral posterior medial thalamus. $J$. Pharmacol. Exp. Ther., 327, 20-31.

Tachibana, T., Endoh, M., Fujiwara, N., \& Nawa, T. (2005) Receptors and transporter for serotonin in Merkel cell-nerve endings in the rat sinus hair follicle. An immunohistochemical study. Arch Histol Cytol, 68, 19-28.

Takeuchi, M.S., Miyaoka, H., Tomoda, A., Suzuki, M., Liu, Q., \& Kitamura, T. (2010) The effect of interpersonal touch during childhood on adult attachment and depression: A neglected area of family and developmental psychology? J. Child Fam. Stud., 19, 109- 
117.

Tzourio-Mazoyer, N., Landeau, B., Papathanassiou, D., Crivello, F., Etard, O., Delcroix, N., Mazoyer, B., \& Joliot, M. (2002) Automated anatomical labeling of activations in SPM using a macroscopic anatomical parcellation of the MNI MRI single-subject brain. Neuroimage, 15, 273-289.

v. Békésy, G. (1957) Neural Volleys and the Similarity between Some Sensations Produced by Tones and by Skin Vibrations. J. Acoust. Soc. Am., 29, 1059.

Vallbo, A.B., Olausson, H., \& Wessberg, J. (1999) Unmyelinated afferents constitute a second system coding tactile stimuli of the human hairy skin. J. Neurophysiol., 81, 2753-2763.

Verrillo, R.T. \& Chamberlain, S.C. (1972) The effect of neural density and contactor surround on vibrotactile sensation magnitude. Percept. Psychophys., 11, 117-120.

Voos, A.C., Pelphrey, K.A., \& Kaiser, M.D. (2013) Autistic traits are associated with diminished neural response to affective touch. Soc Cogn Affect Neurosci, 8, 378-386.

Wang, L., Mullette-Gillman, O. a., Gadde, K.M., Kuhn, C.M., McCarthy, G., \& Huettel, S. a. (2009) The effect of acute tryptophan depletion on emotional distraction and subsequent memory. Soc. Cogn. Affect. Neurosci., 4, 357-368.

Wardle, M.C., Kirkpatrick, M.G., \& de Wit, H. (2014) "Ecstasy” as a social drug: MDMA preferentially affects responses to emotional stimuli with social content. Soc. Cogn. Affect. Neurosci., 9, 1076-1081.

Waterhouse, B.D., Moises, H.C., \& Woodward, D.J. (1986) Interaction of serotonin with somatosensory cortical neuronal responses to afferent synaptic inputs and putative neurotransmitters. Brain Res. Bull., 17, 507-518.

Weber, A.I., Saal, H.P., Lieber, J.D., Cheng, J.-W., Manfredi, L.R., Dammann, J.F., \& Bensmaia, S.J. (2013) Spatial and temporal codes mediate the tactile perception of natural textures. Proc. Natl. Acad. Sci. U. S. A., 110, 17107-17112.

Weiss, S.J., Wilson, P., Hertenstein, M.J., \& Campos, R. (2000) The tactile context of a mother's caregiving: Implications for attachment of low birth weight infants. Infant Behav. Dev., 23, 91-111.

Weltzin, T.E., Fernstrom, J.D., McConaha, C., \& Kaye, W.H. (1994) Acute tryptophan depletion in bulimia: effects on large neutral amino acids. Biol. Psychiatry, 35, 388-397.

Wildgruber, D., Hertrich, I., Riecker, A., Erb, M., Anders, S., Grodd, W., \& Ackermann, H. (2004) Distinct Frontal Regions Subserve Evaluation of Linguistic and Emotional Aspects of Speech Intonation. Cereb. Cortex, 14, 1384-1389.

Williams, J.H.G., Perrett, D.I., Waiter, G.D., \& Pechey, S. (2007) Differential effects of tryptophan depletion on emotion processing according to face direction. Soc. Cogn. Affect. Neurosci., 2, 264-273.

Wood, R.M., Rilling, J.K., Sanfey, A.G., Bhagwagar, Z., \& Rogers, R.D. (2006) Effects of tryptophan depletion on the performance of an iterated Prisoner's Dilemma game in healthy adults. Neuropsychopharmacology, 31, 1075-1084.

Yoshioka, T., Gibb, B., Dorsch, a K., Hsiao, S.S., \& Johnson, K.O. (2001) Neural coding mechanisms underlying perceived roughness of finely textured surfaces. J. Neurosci., 
21, 6905-6916.

Young, S.N. (2013) The effect of raising and lowering tryptophan levels on human mood and social behaviour. Philos. Trans. R. Soc. Lond. B. Biol. Sci., 368, 20110375. 
Table 1: Age, IQ, total plasma tryptophan and mood before and after treatment for both treatment groups

TRP+

TRP-

0 hours $\quad+4$ hours $\quad 0$ hours $\quad+4$ hours

\begin{tabular}{|c|c|c|c|c|}
\hline Age (years) & \multicolumn{2}{|c|}{$22.6(0.78)$} & \multicolumn{2}{|c|}{$24.8(1.62)$} \\
\hline IQ & \multicolumn{2}{|c|}{$96.6(2.19)$} & \multicolumn{2}{|c|}{$98.6(1.61)$} \\
\hline $\begin{array}{l}\text { Plasma TRP } \\
(\mu \mathrm{g} / \mathrm{ml})\end{array}$ & $7.7(0.33)$ & $20.9(0.94)$ & $8.0(0.53)$ & $2.0(0.12)$ \\
\hline POMS TMD & $-10.2(3.85)$ & $-6.21(4.43)$ & $-12.31(2.54)$ & $-7.69(2.52)$ \\
\hline FCPS & $121.64(4.67)$ & $118.14(5.10)$ & $121.56(3.01)$ & $119.56(2.45)$ \\
\hline
\end{tabular}

Comparison of age, total plasma tryptophan concentration (plasma TRP) and mood (profile of mood states total mood disturbance (POMS TMD) and Fawcett-Clark pleasure scale (FCPS) scores are shown) across treatment groups (TRP+: control drink, TRP-: tryptophan depleting drink) and by time (baseline ( 0 hours) and 4 hours post amino acid consumption (+ 4 hours)). Mean values (with SE) are presented. No significant differences were identified, except for total plasma tryptophan concentrations where plasma tryptophan increased significantly in the TRP + group $(p<0.001)$ and decreased significantly in the TRP-group $(p<0.001)$ at +4 hours compared to baseline. Plasma tryptophan concentrations were comparable between groups at baseline $(p=0.743)$, but significantly higher in the TRP+ than the TRP-group 4 hours after drink consumption $(p<0.001)$. 
Table 2: fMRI significant interaction effects of treatment, texture and location

\begin{tabular}{llllll}
\hline & $\begin{array}{l}\text { Cluster size } \\
\text { (voxels) }\end{array}$ & $\begin{array}{l}\text { Peak } \boldsymbol{p} \\
\text { (FDR } \\
\text { corrected) }\end{array}$ & $\begin{array}{l}\text { Peak } \boldsymbol{\chi}^{\mathbf{2}} \text { - } \\
\text { value }\end{array}$ & $\begin{array}{l}\text { Coordinates } \\
(\mathbf{X ~ Y ~ Z ) ~}\end{array}$ & Location \\
\hline $\begin{array}{l}\text { Treatment x Texture x } \\
\text { Location }\end{array}$ & 203 & $<0.001$ & 34.74 & $44-2753$ & Right SI \\
\hline Treatment x Location & 273 & $<0.001$ & 34.36 & $44-2749$ & Right SI \\
& 16 & 0.016 & 13.76 & $-3727-18$ & Left OFC (IFG) \\
\hline Location x Texture & 495 & $<0.001$ & 34.77 & $47-2653$ & Right SI \\
& 47 & 0.003 & 16.37 & $-44-2942$ & Left SI \\
& 14 & 0.003 & 16.07 & $-37-2453$ & Left SI \\
\hline & 10 & 0.016 & 12.14 & $60-1311$ & Right SII (OP4) \\
\hline
\end{tabular}

Abbreviations: IFG - inferior frontal gyrus, OFC - orbitofrontal cortex, SI - primary somatosensory cortex, SII - secondary somatosensory cortex, OP4 - parietal operculum area 4 . MNI coordinates are stated. 
Table 3: Brain regions for which a significant effect of location was identified

\begin{tabular}{|c|c|c|c|c|c|}
\hline & $\begin{array}{l}\text { Cluster size } \\
\text { (voxels) }\end{array}$ & $\begin{array}{l}\text { Peak } p(\text { FDR } \\
\text { corrected })\end{array}$ & $\begin{array}{l}\text { Peak } \chi^{2}- \\
\text { value }\end{array}$ & $\begin{array}{l}\text { Coordinates } \\
(\text { X Y Z) }\end{array}$ & Location \\
\hline \multirow[t]{4}{*}{ Finger $>$ Arm } & 681 & $<0.001$ & 46.51 & $46-2449$ & Right SI \\
\hline & 285 & $<0.001$ & 21.82 & $-44-2942$ & Left SI \\
\hline & 65 & 0.003 & 15.15 & $49-1714$ & SII (OP1) \\
\hline & 18 & 0.017 & 10.87 & $42-40$ & Right Mid-Insula \\
\hline \multirow[t]{3}{*}{ Arm $>$ Finger } & 40 & 0.003 & 14.95 & $-37-2453$ & $\begin{array}{l}\text { Left SI (arm less } \\
\text { deactivation than } \\
\text { fingers) }\end{array}$ \\
\hline & 8 & 0.013 & 11.5 & 81025 & $\begin{array}{l}\text { Anterior } \\
\text { Cingulate }\end{array}$ \\
\hline & 5 & 0.024 & 10.1 & $31-2018$ & $\begin{array}{l}\text { Right Posterior } \\
\text { Insula (Ig2) }\end{array}$ \\
\hline
\end{tabular}

Abbreviation: SI - primary somatosensory cortex, SII - secondary somatosensory cortex, OP1 parietal operculum area $1, \operatorname{Ig} 2$ - granular insula area 2. MNI coordinates are stated. 
Table 4: main effect of touch to the forearm and fingers compared to rest

\begin{tabular}{|c|c|c|c|c|c|}
\hline & $\begin{array}{l}\text { Cluster size } \\
\text { (voxels) }\end{array}$ & $\begin{array}{l}\text { Peak } p(\text { FDR } \\
\text { corrected) }\end{array}$ & $\begin{array}{l}\text { Peak z- } \\
\text { value }\end{array}$ & $\begin{array}{l}\text { Coordinates } \\
\text { (X Y Z) }\end{array}$ & Location \\
\hline \multirow{5}{*}{$\begin{array}{l}\text { Forearm }> \\
\text { rest }\end{array}$} & 251 & 0.007 & 4.63 & $-3939-11$ & Left OFC (IFG) \\
\hline & 190 & 0.007 & 4.61 & $-61-2439$ & Left SII \\
\hline & 52 & 0.007 & 4.51 & $37-1518$ & $\begin{array}{l}\text { Right Posterior } \\
\text { Insula }\end{array}$ \\
\hline & 47 & 0.008 & 3.90 & $58-1725$ & Right SII \\
\hline & 9 & 0.027 & 3.19 & $24-4070$ & Right SI \\
\hline \multirow{8}{*}{$\begin{array}{l}\text { Finger }> \\
\text { rest }\end{array}$} & 1257 & $<0.001$ & 7.39 & $46-2653$ & Right SI \\
\hline & 733 & $<0.001$ & 5.78 & $-62-2035$ & Left SI \\
\hline & 247 & $<0.001$ & 4.19 & $-4446-4$ & Left OFC (IFG) \\
\hline & 64 & 0.001 & 3.81 & $\begin{array}{llll}-35 & -6 & 11\end{array}$ & Left mid-insula \\
\hline & 40 & 0.003 & 3.55 & $4634-11$ & Right OFC (IFG) \\
\hline & 5 & 0.012 & 3.01 & -46514 & Left SII \\
\hline & 7 & 0.016 & 2.87 & $-2630-14$ & Left OFC (IFG) \\
\hline & 9 & 0.017 & 2.87 & $-5327-4$ & Left OFC (IFG) \\
\hline
\end{tabular}

Abbreviations: IFG - inferior frontal gyrus, OFC - orbitofrontal cortex, SI - primary somatosensory cortex, SII - secondary somatosensory cortex. MNI coordinates are stated. 


\section{Figure Legends}

Figure 1: Hedonic ratings for the soft, medium and coarse brushes when applied to the forearm and fingers. Error bars represent $95 \%$ confidence intervals. A significant interaction of brush texture with location was identified $(p<0.001)$. For touch to both the forearm and fingers, the soft brush was more pleasant than the medium brush, which was more pleasant than the coarse brush ( $p_{S}<0.001$ for all comparisons). The soft brush was more pleasant when applied to the forearm compared to the fingers $\left(p_{S}=0.022\right)$, the medium brush was more pleasant when applied to the fingers than the forearm $\left(p_{S}=0.001\right)$ and the coarse brush was more unpleasant when applied to the forearm than the fingers $\left(p_{S}=0.022\right) .{ }^{*} p<0.05, * * p<0.01$

Figure 2: Significant interaction of treatment with location in left OFC (MNI -37 25 -18). Error bars represent $95 \%$ confidence intervals. In the control (TRP+) condition, touch to the forearm induced significantly greater activation than touch to the fingers. Following tryptophan depletion (TRP-), no significant difference in BOLD response was identified following touch to the fingers compared to the forearm. ${ }^{*} p_{\mathrm{FDR}}<0.05$

Figure 3: A significant interaction of treatment group with stimulus texture and touch location was identified in right somatosensory cortex (MNI: 44 -27 53). Error bars represent $95 \%$ confidence intervals. In the control (TRP+) condition for touch to the fingers, texture discrimination was significant, with the coarse texture response significantly greater than the soft texture. This effect was not present following tryptophan depletion (TRP-), where no texture discrimination was identified following touch to the fingers. Additionally, for each texture and in both treatment groups, touch to the fingers produced significantly greater 
response than to the forearm. Abbreviations: $\mathrm{SB}$ - soft brush, $\mathrm{MB}$ - medium brush, $\mathrm{CB}-$ coarse brush. ${ }^{*} p_{\mathrm{FDR}}<0.05$

Figure 4: Effect of touch location. Error bars represent $95 \%$ confidence intervals. CT-targeted forearm touch induced significantly greater BOLD response than CT non-targeted touch to the fingers in the anterior cingulate (MNI 810 25) (A) and right posterior insula (Ig2) (MNI 31 20 18) (B). A significantly greater BOLD response was seen in secondary somatosensory cortex (SII), parietal operculum area 1 (OP1) (MNI 49 -17 14) (C) and right mid-insula (MNI $42-4$ 0) (D) following touch to the fingers compared to the forearm. ${ }^{*} p_{\text {FDR }}<0.05$ 


\section{Graphical abstract text}

C-tactile afferents (CTs) are present in hairy skin, responding to caress-like touch and hypothesised to provide the neural substrate for affective touch. Acute tryptophan depletion (TRP-) and fMRI were used to investigate the role of serotonin in central responses to CT vs non-CT touch. Findings implicate a role for serotonin in differentiating between $\mathrm{CT} /$ non-CT touch, providing a potential mechanism underlying altered touch responses in psychiatric disorders such as depression and autism. 